\title{
Confort Térmico: \\ Un sistema aislante para la vivienda alto andina fabricado con materiales reciclados
}

Thermal Comfort:
An insulation system for alto andina
housing made with recycled materials

DOI: 10.179 81/mod.arq.cuc.18.1.2017.04

Artículo. Fecha de recepción: 25/08/2018 Fecha de aceptación: 27/10/2017

\author{
César Moncloa Guardia \\ Universidad Continental Sede Huancayo (Perú) \\ cesarmoncloa@gmail.com
}

Para citar este artículo:

Moncloa, C. (2017). Confort Térmico: Un sistema aislante para la vivienda Alto Andina fabricado con materiales reciclados. MODULO ARQUITECTURA-CUC, vol. 18, no. 1, pp. 73-90. DOI: 10.17981/mod.arq.cuc.18.1.2017.04

\section{Resumen}

El presente trabajo presenta una experiencia investigativa en el ámbito de la bioclimática y las condiciones adecuadas para el confort término en zonas andinas de Perú. Con el objetivo de reducir el drama de las muertes por enfermedades asociadas al frío en las zonas Alto Andinas del Perú en épocas de heladas, este proyecto es una apuesta por la investigación aplicada desde la universidad. Desdibujando la brecha entre en el salón y la realidad¹.

Palabras claves: Confort térmico, sistemas aislantes, material reciclado, Los Andes,

\begin{abstract}
This research presents a materialized project experience in the field of bioclimatics and the adequate conditions for the Thermal Comfort in Andean areas of Peru. With the aim of reducing the drama of deaths from illnesses associated with cold in the alto andinas areas of Peru in times of frost, this project is a commitment to applied research from the university. Blurring the gap between the living room and reality.
\end{abstract}

Keywords: Thermal comfort, insulating systems, recycled material, Territory of the Andes.

${ }^{1}$ Desarollada por el arquitecto César Moncloa y alumnos de la catedra de Acondicionamiento y Servicios I de la Universidad Continental Sede Huancayo. 


\section{INTRODUCCIÓN}

El proyecto "CONFORT TÉRMICO" persigue estratégicamente, a partir de los proceso de investigación aplicada, planteados desde la interacción con los estudiantes de arquitectura, cambiarle el rostro a la pobreza extrema en nuestro país (Perú). Desarrollando un sistema aislante que impida que la temperatura descienda dramáticamente al interior de las viviendas, cuyo costo sea cero, que pueda ser implementado por cualquier persona, en todas las localidades donde se lo necesite e instalado en cualquier tipología de vivienda rural.

El proyecto pretende despertar en el alumno la conciencia ética al estar siendo educado para resolver los problemas de su comunidad, entendiendo a la realidad como un sistema que debe mejorar.
Para lograr este objetivo se reducen las horas teóricas, se discuten los problemas sociales, económicos y territoriales a los que se enfrentan. El salón poco a poco se va convirtiendo en un taller, las verdades se reemplazan por hipótesis y la teoría no se aprende, se va construyendo, experimento tras experimento (Figs. 1a y 1b). En un sistema convencional de enseñanza se valora la competitividad entre los estudiantes, en este proyecto lo que se busca es la evolución por colaboración. En ese sentido, el salón debe dejar de ser ese espacio convencional que valora la memoria y la competitividad, para convertirse en un taller de investigación colaborativa.

El Proyecto denominado "CONFORT TÉRMICO", es un sistema creado por el arquitecto César Moncloa junto a sus alumnos de Cátedra de Acondicionamiento

Fig. 1a. Salón de Taller, alumnos de la catedra de Acondicionamiento y Servicios I de la Universidad Continental Sede Huancayo.

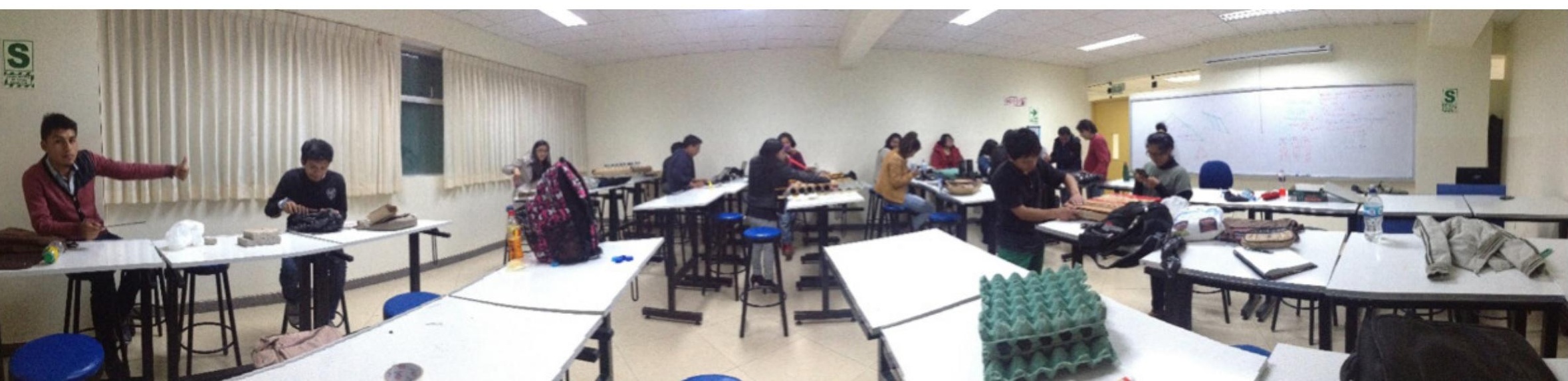


y Servicios I de la Universidad ContinentalSede Huancayo, con el objetivo de reducir el drama de las muertes por enfermedades asociadas al frío en las zonas alto andinas del Perú en épocas de heladas. Al ser un proyecto que se hace tangible mediante la construcción, se debieron analizar con detenimiento los materiales a utilizar, tanto por sus propiedades térmicas como por el costo, que debía ser prácticamente nulo. Se enfocaron los problemas de la contaminación ambiental por desechos de plástico como una oportunidad, mirando la basura como una gran fuente de materiales, muy económicos y alternativos de construcción para el futuro (con una capacidad de desarrollo en nuevos mercados). Se emplearon botellas recicladas de plástico por su acceso prácticamente a un suministro (lamentablemente) ilimitado y gratuito de material para nuestras investigaciones (Fig. 2).
Para darnos una idea del potencial que esto representa, según el Ministerio del Ambiente (2014) solo en la región Junín, en la sierra central del Perú, se producen 479 toneladas de basura por día, contaminando las cuencas naturales de los ríos más importantes de toda la región, haciendo clara la necesidad que hay de plantear una solución a este problema medioambiental. Otro objetivo propuesto a un largo plazo consiste en impulsar el mercado del reciclaje, reenfocando la economía de materiales para que las botellas sean valiosas aun después de su uso primario, eliminando el concepto de basura y cerrando el círculo de la economía de los materiales de un sistema lineal a uno cerrado, beneficiando de esta manera a las empresas al crear productos con una vida útil mucho mayor.

Fig. 1b. Detalle materiales de la catedra de Acondicionamiento y Servicios I de la Universidad Continental Sede Huancayo.

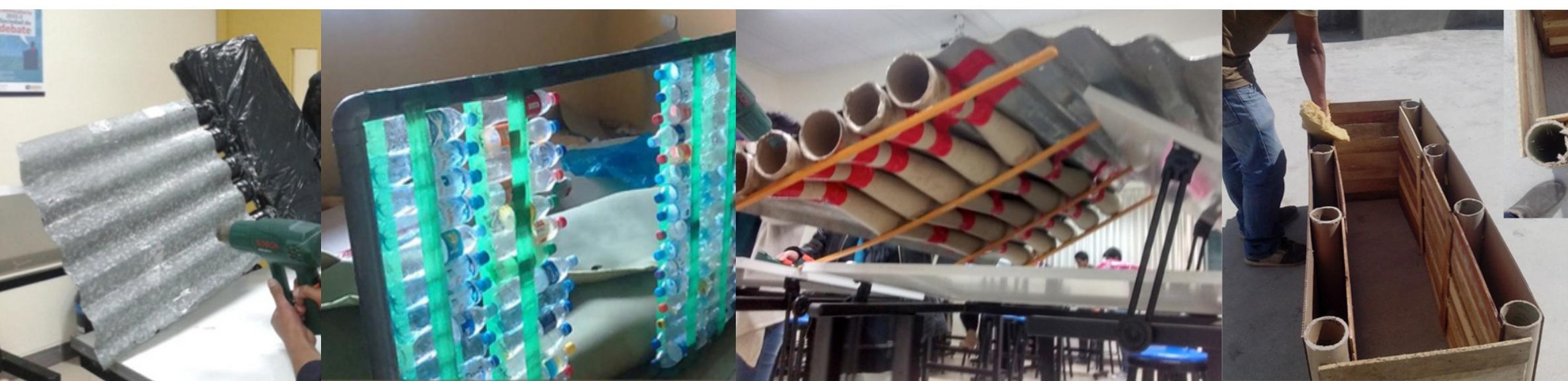




\section{0 - Tha}

Fig. 2. Profesor César Moncloa y alumnos de la Catedra de Acondicionamiento Ambiental I, de la Universidad Continental, (mayo 2016). "Se reciclaron en un par de meses 14.000 botellas" Fuente: elaboración propia.

\section{AREIUTME}

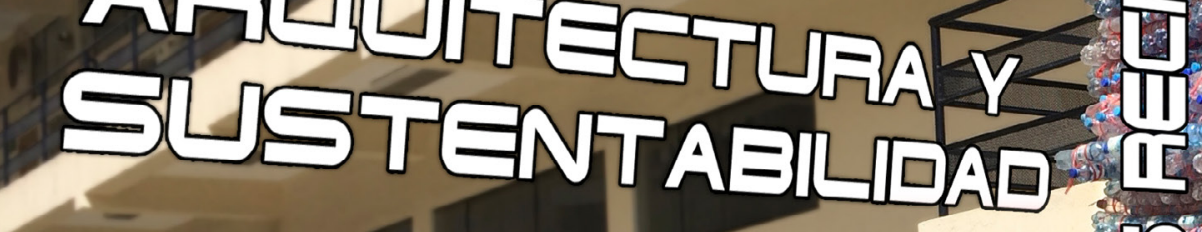

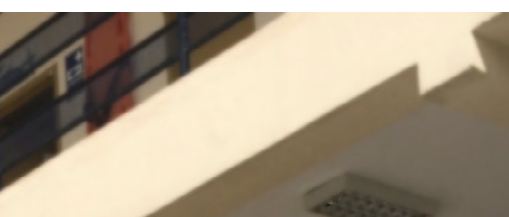

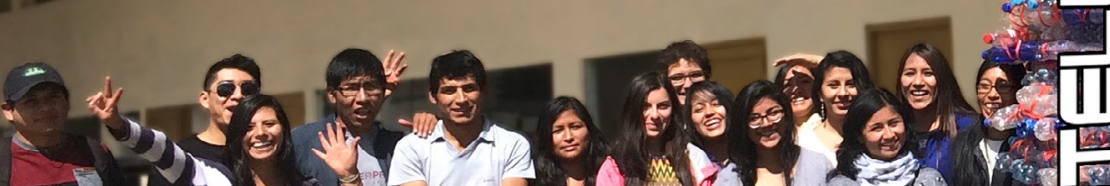
a)

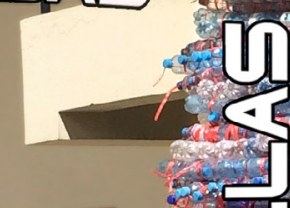

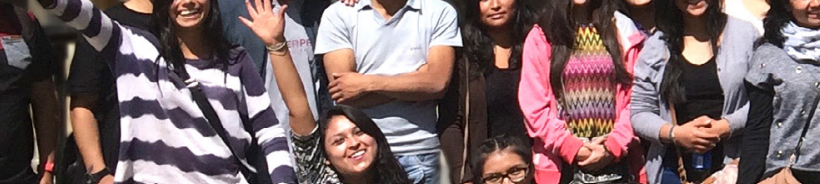

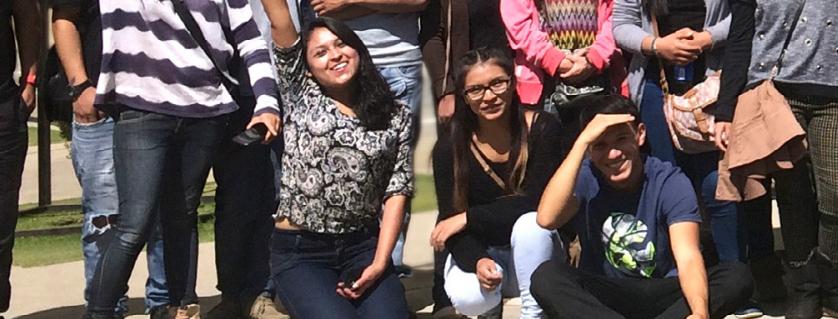

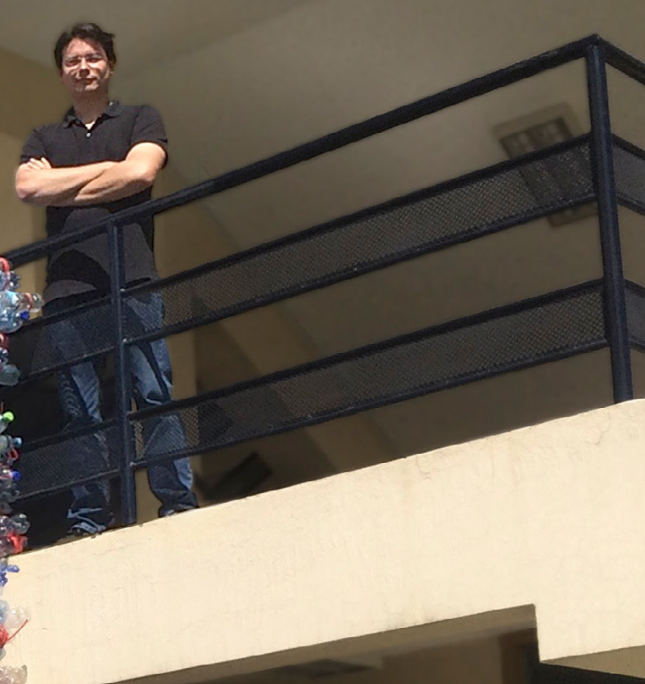




\section{Las Zonas alto andinas en el Perú}

En el Perú, las zonas que presentan la mayor tasa de mortalidad por enfermedades asociadas al frío, curiosamente son también aquellas que reciben una intensa radiación solar. Perú es un territorio muy particular, al contener 28 de los 32 climas que hay en todo el mundo. Las zonas alto andinas a pesar del intenso frío que presentan en las madrugadas son zonas tropicales, lo que condiciona una marcada verticalidad del movimiento aparente del sol, lo que provoca una de las radiaciones solares más intensas del mundo sobre esta región (Servicio Nacional de Meteorología e Hidrología-SENAMHI, 2003) siempre y cuando el cielo este despejado, como es el caso en la temporada de invierno. Sin embargo la gran altitud en la que se encuentra, condiciona temperaturas muy bajas (Fig. 3). Estos factores hacen que en invierno tenga días con una intensa radiación solar y noches extremadamente frías, donde las temperaturas suelen estar por debajo de los $0^{\circ} \mathrm{C}$; este invierno tan particular de noches gélidas y días de intensa radiación solar, puede ser aprovechado para diseñar sistemas pasivos de climatización en las viviendas, que capten esa enorme energía gratuita en forma de calor y la conserven para hacer más confortables las madrugadas de invierno.

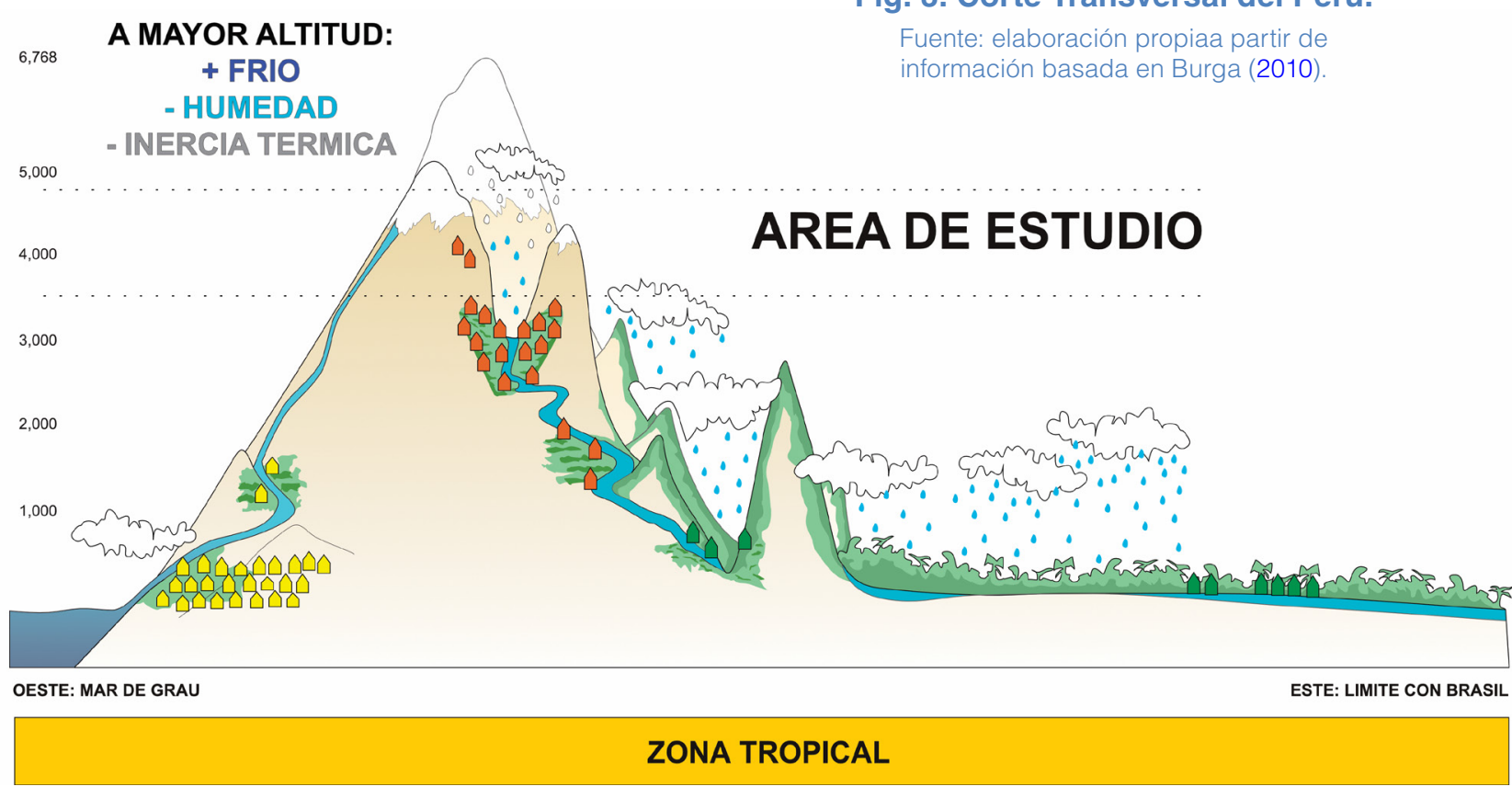

ig. 3. Corte Transversal del Perú. información basada en Burga (2010). 



\section{Las Heladas}

Decenas de peruanos mueren por enfermedades asociadas al frío en las zonas alto andinas, de la misma forma, en la misma época del año y en las mismas circunstancias, año tras año. Según el SENAMHI las infecciones respiratorias y neumonías contraídas a causa de las heladas fueron las responsables de la muerte de 367 niños en el 2012 (Cadillo, 2013) y de acuerdo al Centro de Operaciones en Emergencia Nacional-COEN (2016) las bajas temperaturas afectaron la salud de más de 930 mil personas en el 2016. Las heladas son un fenómeno natural recurrente que se presenta en las madrugadas de otoño e invierno, cuando la temperatura desciende bruscamente por debajo de los $0^{\circ} \mathrm{C}$ hasta los $-15^{\circ} \mathrm{C}$ en promedio; según el "Plan Multisectorial ante Heladas 2017" (Decreto Supremo N ${ }^{\circ}$ 019-2017-PCM, 2017), la temperatura más baja registrada fue de $-28.2^{\circ} \mathrm{C}$. Las zonas afectadas estan comprendidas entre los 3.500 y 5.000 metros sobre el nivel del mar en zonas donde la población residente en su mayoría es predominantemente de extrema pobreza. Se dedican a la agricultura y a la crianza de camélidos sudamericanos, y se agrupan en pequeñas y dispersas comunidades donde las heladas atentan contra su salud, principalmente niños y adultos mayores, así como también afecta a su desarrollo económico, concretamente por la perdida de cultivos locales y la muerte de animales (Fig. 4). Por sus condiciones territoriales de difícil acceso y topografía escarpada es dificil la presencia del estado en estas zonas alejadas del país.

\section{La Vivienda alto andina}

Son espacios muy precarios, construidos con los materiales más económicos o aquellos que se encuentren a la mano, de configuración muy dispersa con "habitaciones de planta rectangular, dispuestas de manera libre alrededor de un espacio central que sirve de elemento de comunicación, con sus corrales abiertos" (Burga, 2010). Los muros pueden ser de adobe, tapial o piedra, lo que garantiza un muro de no menos de $40 \mathrm{~cm}$ con un alto grado de inercia térmica, muy bueno para conservar el calor, sin embargo, a veces presentan huecos que permiten la entrada de aire frío del exterior (Figs. 5a y 5b). Los techos frecuentemente tienen una estructura de madera a dos aguas, antiguamente revestidos con ichu (paja), tejas o una combinación de las anteriores, que proveen una capa aislante de cierta efectividad protegiendo la casa del frío, lluvias y granizo.

La mayoría de estos techos están muy descuidados, llegando a presentar pequeños huecos y filtraciones que hacen imposible conservar el calor, esto se agrava cuando llueve o graniza, al caer directamente el agua o hielo en los dormitorios, muchas veces sobre las camas de sus ocupantes. Actualmente la sabiduría ancestral de construcción se está perdiendo rápidamente y los techos son remplazados por las fáciles en instalar y económicas planchas de calamina galvanizada, que si bien impermeabilizan el techo, su resistencia al calor es prácticamente nula, haciéndola desde el punto de vista del confort térmico la peor opción para combatir las heladas. 
Fig. 5a. Típica vivienda Alto Andina dedicada a la crianza de alpaca, Comunidad de Siusa en Junín.

Fuente: elaboración propia.

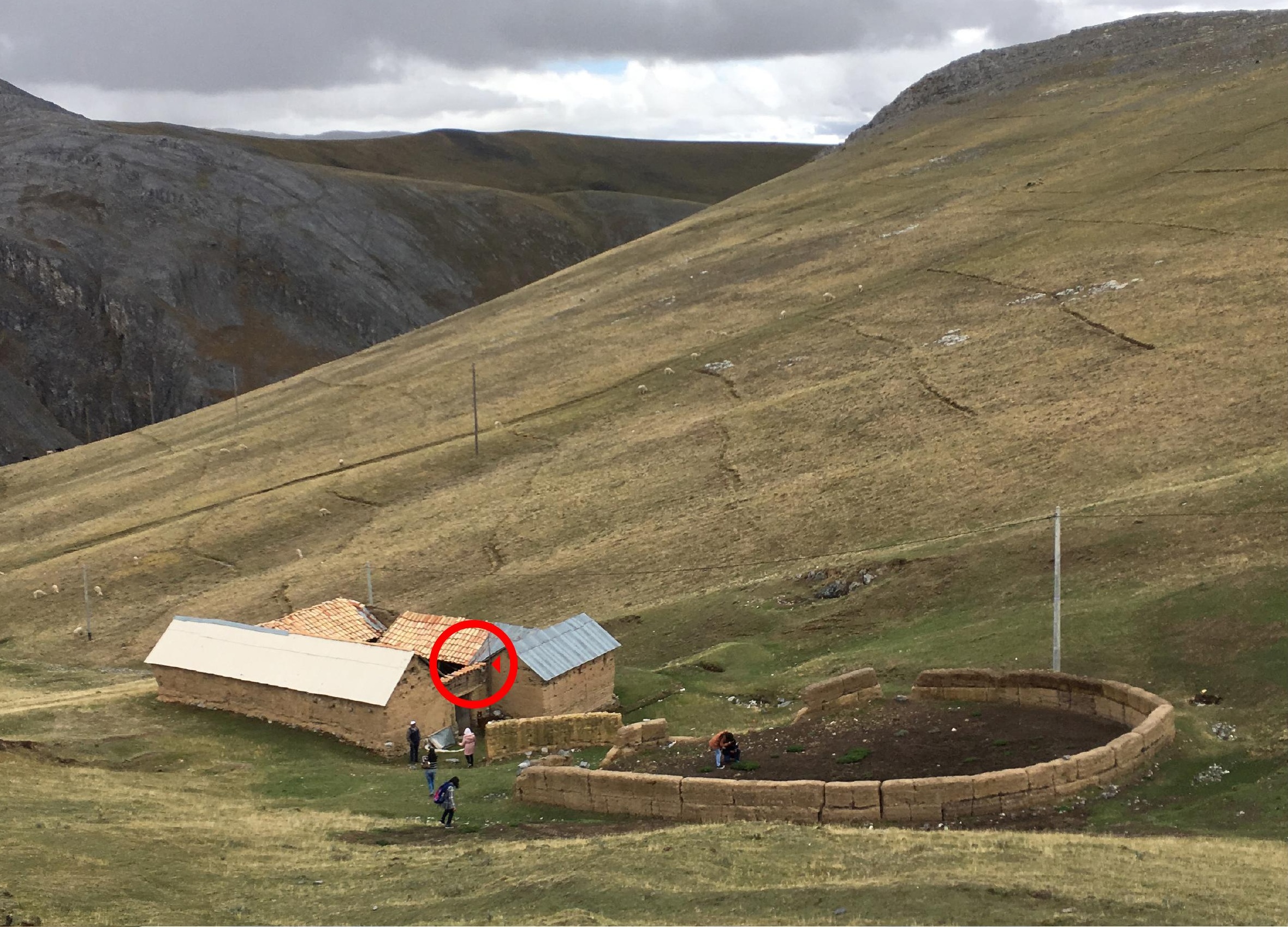


Los pisos no tienen tratamiento, es la misma tierra apisonada del suelo permitiendo el ingreso de humedad y salida del calor. Las ventanas de vidrio aunque pequeñas en área dejan escapar mucho calor, debido a que los marcos no están herméticamente sellados al muro y el vidrio casi no presenta resistencia al calor. Las puertas de madera o a veces una simple plancha de calamina presentan el mismo problema. Estas características hacen a la vivienda alto andina en su conjunto un lugar muy vulnerable a las heladas con rangos de temperatura interior fuera de la zona de confort las 24 horas del día.

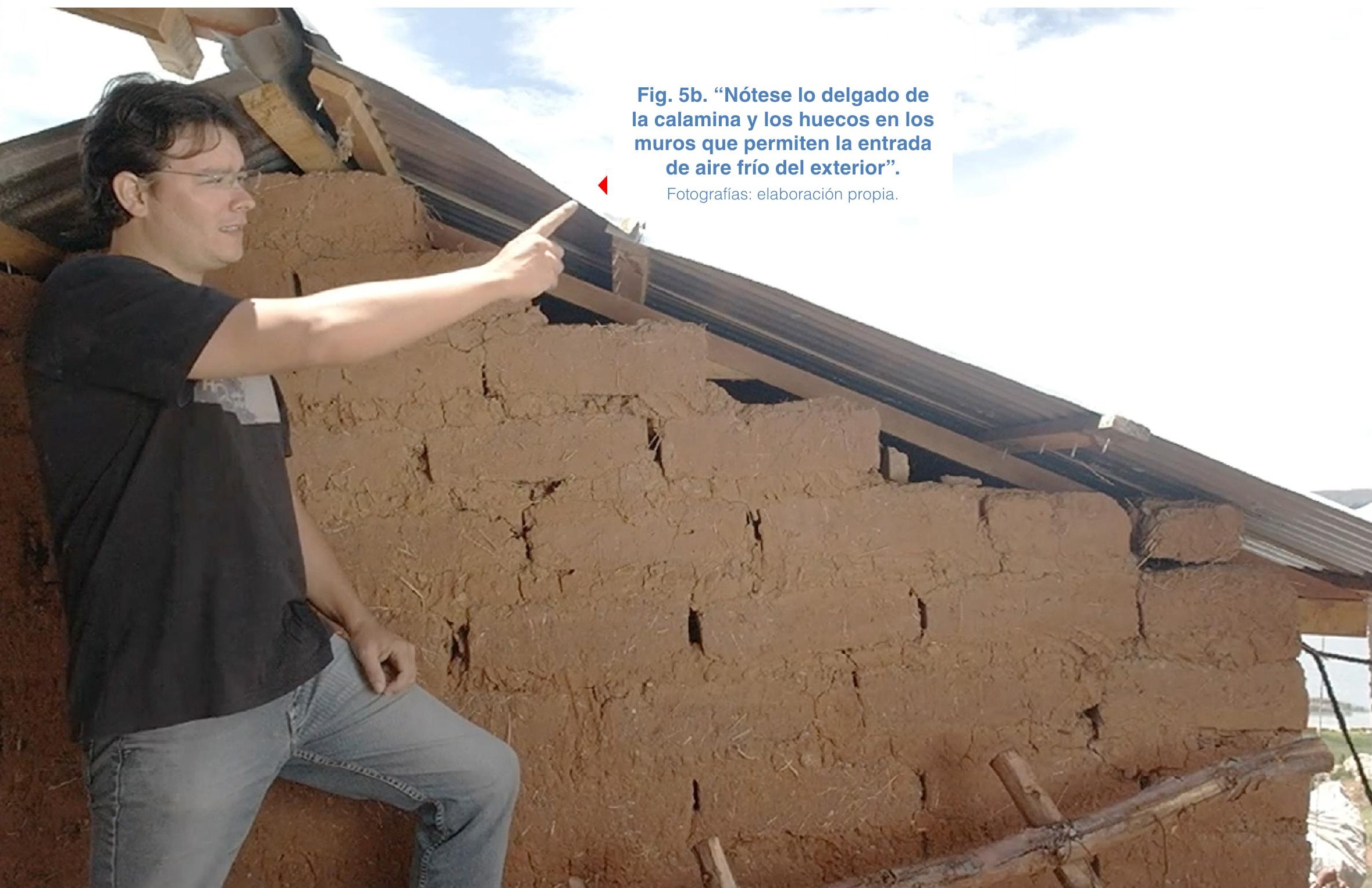




\section{Confort Térmico: ¿Cómo fuciona este Sistema?}

El aire es el mejor aislante, no solo por tener una conductividad térmica muy baja, que dificulta mucho el paso del calor por conducción, sino que además es gratis, requisito indispensable para este proyecto; y para que este aislante funcione debe estar encapsulado, haciendo imperativa la necesidad de un material muy económico que pueda contener aire de forma lo más herméticamente posible. La botella reciclada de PET de $750 \mathrm{ml}$. se convirtió rápidamente en la opción más viable, y existe un mercado de reciclaje que está en capacidad de suministrar miles de botellas en cualquier momento. Y su diseño, afortunadamente tiene como propósito ser un contenedor.

Un kilogramo de botellas recicladas de $750 \mathrm{ml}$. cuesta 0.80 soles (1/4 de dólar) y contiene aproximadamente unas 30 botellas. Pero en los depósitos municipales el plástico no está disgregado, el plástico de todo tipo está mezclado; envases de helados, de yogurt, botellas de todo tipo y tamaño, etc. Se deben escoger manualmente las botellas necesarias, en un lento proceso que puede necesitar varias horas. $Y$ es imperativo que la basura este disgregada para ser distribuida rápidamente a las plantas recicladoras.

Este sistema de climatización es bastante simple, a cada botella plástica se le corta la base para luego empalmarla con otra y hasta crear tubos de $1.8 \mathrm{~m}$. de largo por 0.8 de ancho, donde cada botella contiene un compartimiento de aire estancado (Fig. 6). Un filamento de rafia (cuerda de plástico muy común en el Perú) amarrada al interior de las botellas de este tubo garantiza la cohesión por compresión. La rafia es un material muy resistente, fácil de encontrar en cualquier comunidad del alto andino y bastante económica, por un dólar se pueden implementar para este sistema en 203 viviendas dependiendo del volumen del espacio.

Aproximadamente cada 10 tubos dispuestos uno al costado del otro, se rigidizan a través de dos hileras de tubos adicionales, dispuestos en sentido contrario uno al inicio y otro al final, formando lo que se denomina una "parrilla", para la cual se necesitan 140 botellas con un peso aproximado de 4 $\mathrm{Kg}$. Para aislar el techo de un dormitorio promedio de $25 \mathrm{~m}^{2}$ en una vivienda alto andina, se necesitan 18 parrillas con un costo total de 58 soles. Para tener una idea de como cubrir este mismo techo con calaminas, como se mencionó anteriormente es "de lejos" el material más barato y el menos eficiente en términos de aislante térmico, con un costo de unos 300 soles. Finalmente las parrillas se amarran al techo hacia el interior de la vivienda, agregando una capa de material aislante que disminuye las pérdidas de calor durante las heladas, lo mismo que con las puertas y las ventanas (Fig. 7). 


\section{Implementacion e impacto generado}

El ADN de la Universidad Continental se basa en crear impacto social positivo. A la fecha son 25 familias en las zonas más pobres y susceptibles a la presencia de heladas, las que se han beneficiado con este sistema. La financiación fue cubierta íntegramente por la cátedra y se han reciclado 120,000 botellas de plástico. Las comunidades intervenidas son: en la región Junín; las comunidades de Río Molino, Distrito de Pomacancha, las provincias de Jauja y Siusa, Distrito de Quilcas y la provincia de Huancayo. Y en la región de Puno; la comunidad de Luquina, ubicada en la península de Chucuito, a orillas del lago Titicaca, al sur de la capital de la región (Fig. 8).

Fig. 8. Comunidades Intervenidas. Infografía y Fotografías Fotografía: elaboración propia.
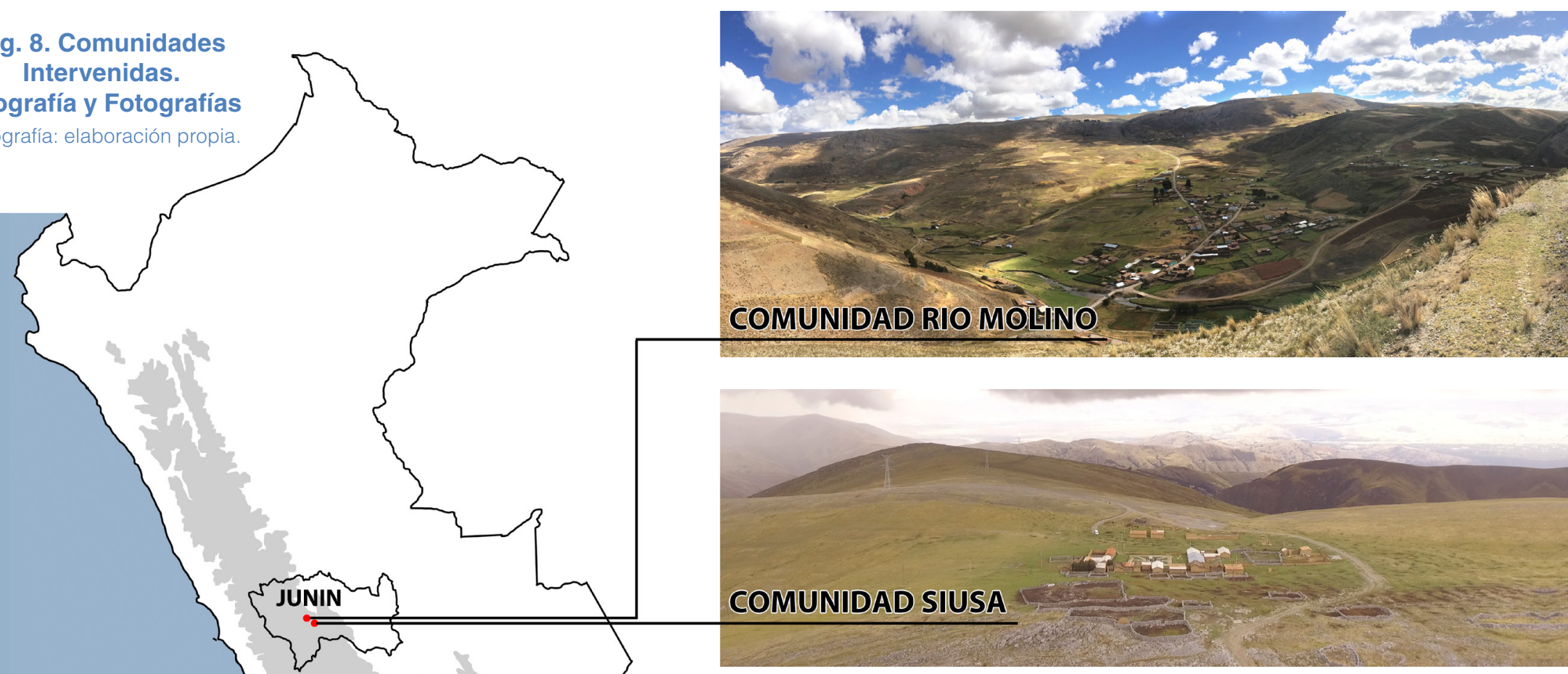

COMUNIDAD SIUSA
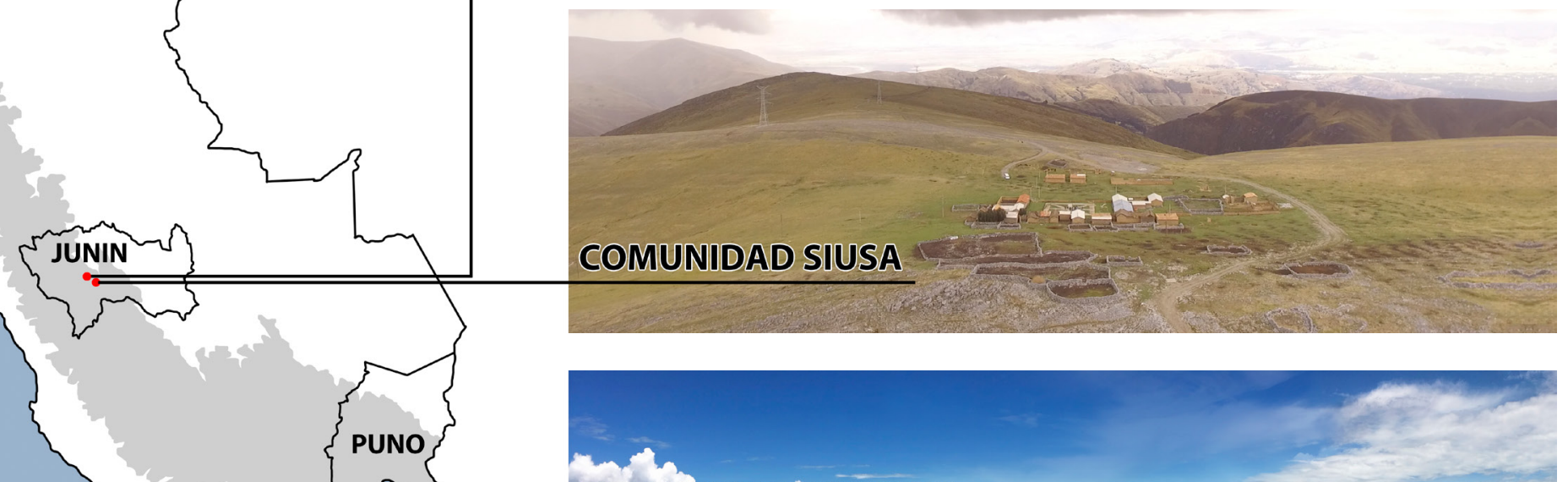
CON TEMPERATURAS MINIMAS POR DEBAJO DE $0^{\circ} \mathrm{C}$

\section{COMUNIDAD LUQUINA}

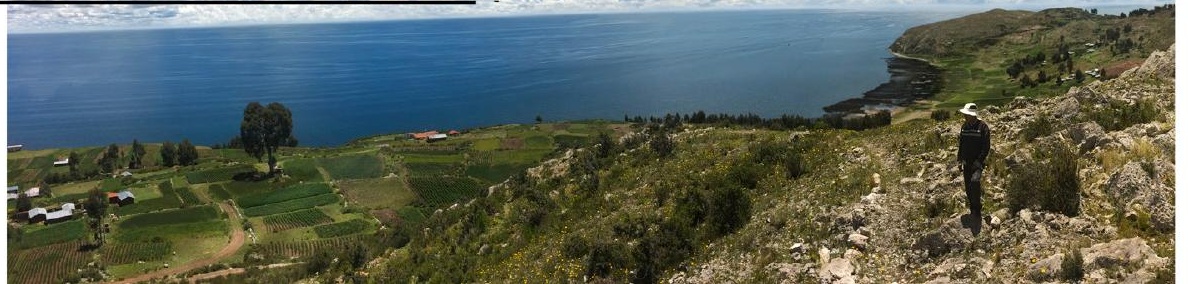


El sistema se mejora continuamente semestre tras semestre; para probar su efectividad se realizan mediciones térmicas a intervalos de una hora desde las 12 de la noche hasta las 5 de la mañana en las viviendas escogidas, primero en los espacios sin tratar, y luego de instalado el sistema, se vuelven a medir para comparar los datos de la vivienda con y sin el sistema de "CONFORT TÉRMICO". En ambos casos estas mediciones se realizan a lo largo de una semana (Fig. 9).

En la actualidad, "CONFORT TÉRMICO" ha logrado una mejora de $2^{\circ} \mathrm{C}$ a $3^{\circ} \mathrm{C}$, comparado con las viviendas antes de instalado el sistema, tal como se presenta en la Fig. 10. La meta actual es aumentar la efectividad hasta llegar a los $5^{\circ} \mathrm{C}$ o $6^{\circ} \mathrm{C}$, para lo cual estamos termo formando la botella reciclada, y así lograr una sección cuadrada que se pueda modular eficientemente, reduciendo los puentes térmicos resultantes de juntar dos cilindros, además de aumentar de 1 capa de material a 3 . Todo ello gracias a que el material del que estan elaboradas las botellas es PET, un plástico que puede variar fácilmente de forma con un molde al aplicársele calor. En agosto de 2017 se aplicara esta variante para comprobar su efectividad.
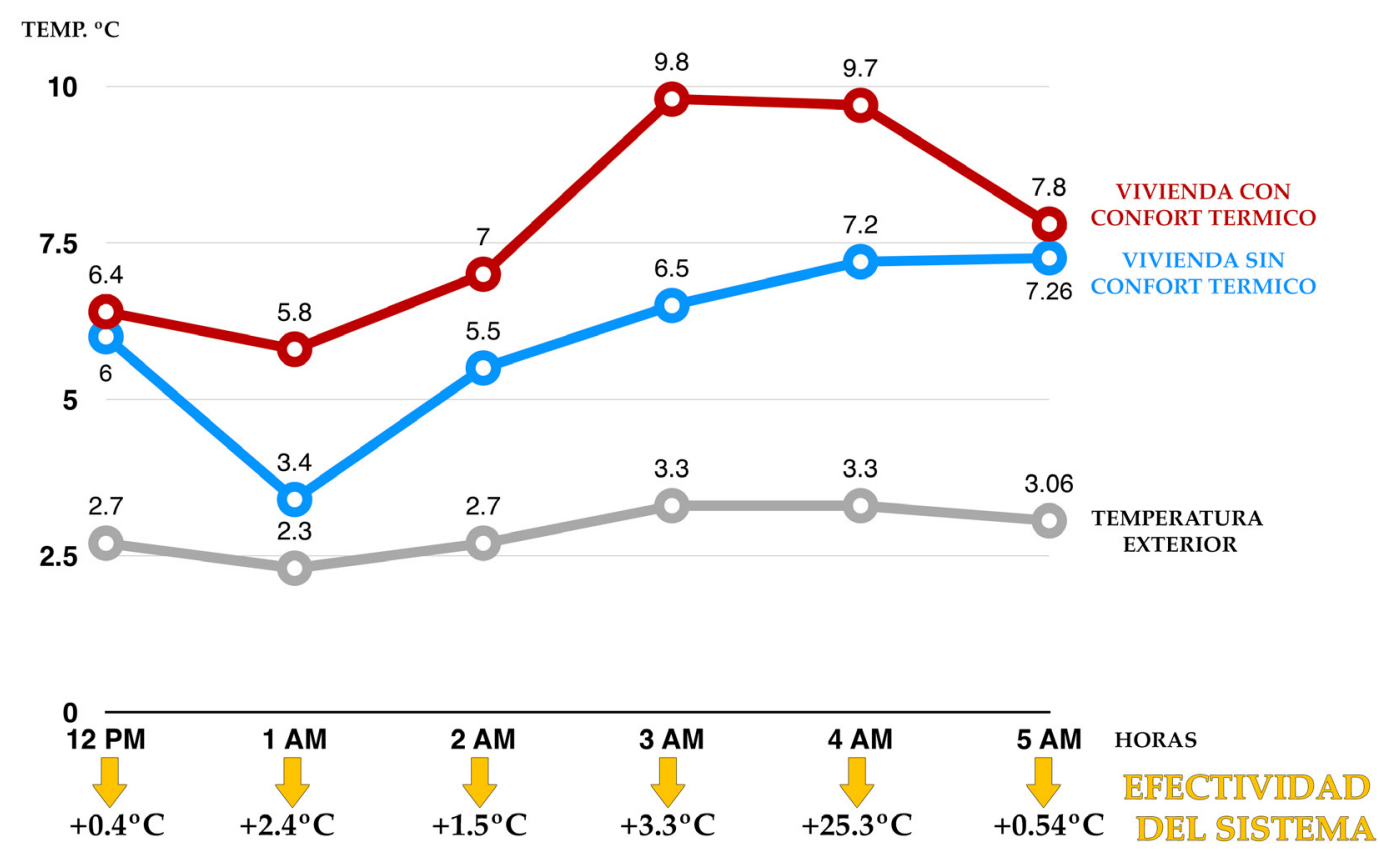

Fig. 10. Cuadro resume de las mediciones en la comunidad de SIUSA, elevando la temperatura interior en $3.3^{\circ} \mathrm{C}$ a las $3 \mathrm{AM}$. 


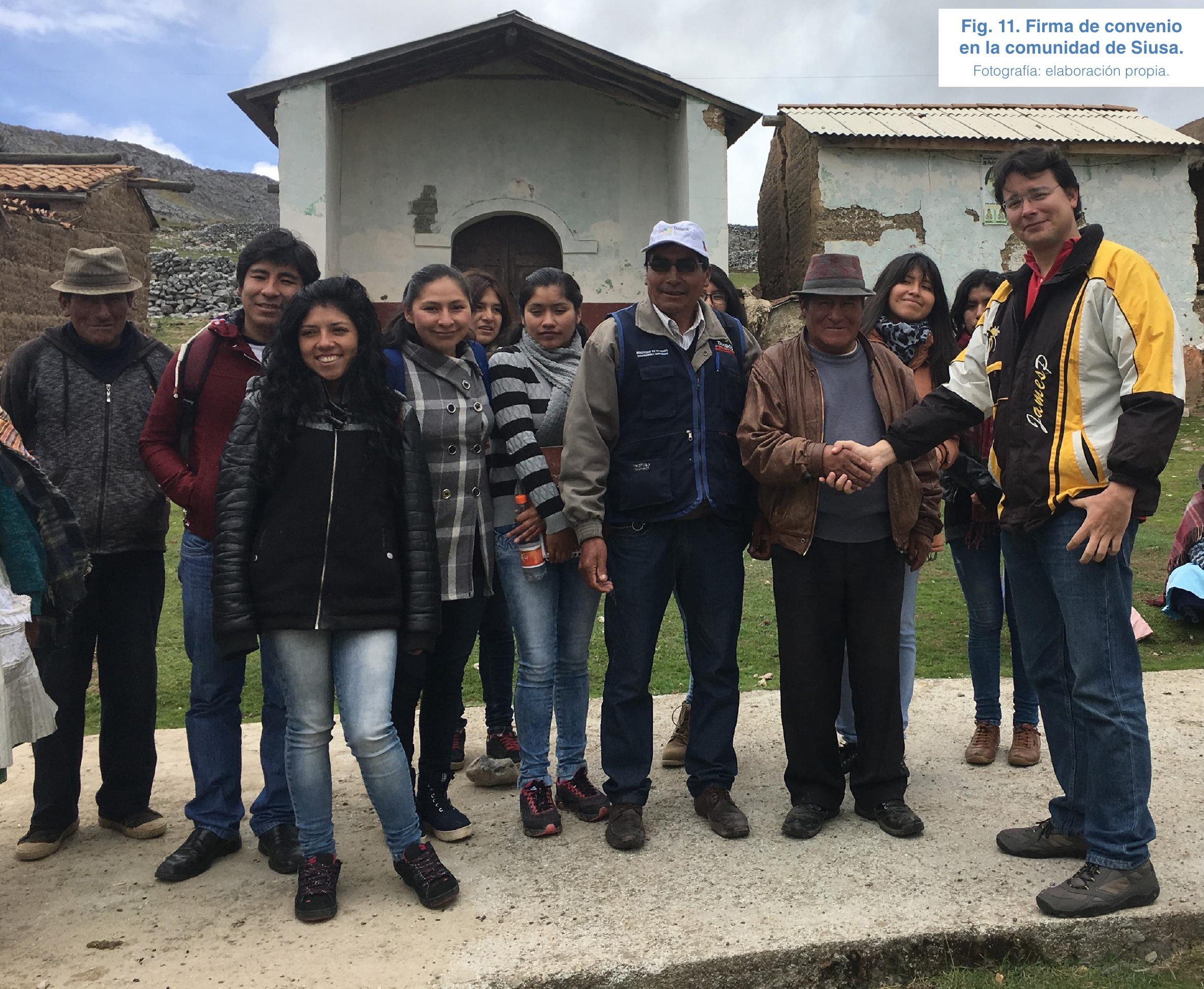


El Ministerio de Vivienda Construcción y Saneamiento en la división Programa Nacional de vivienda rural TAMBOS, se ha interesado en la potencialidad de masificación del sistema de "CONFORT TÉRMICO", convirtiéndose en un aliado estratégico en conjunto con la Universidad Continental para replicar los resultados y conseguir más beneficiarios. En la Fig. 11, podemos observar la firma de un convenio en la comunidad de Siusa el 4 de noviembre del 2016 entre el Ministerio de Vivienda, la escuela de Arquitectura de la Universidad Continental y el presidente de la comunidad con la finalidad de implementar a 6 viviendas el sistema de "CONFORT TÉRMICO".

\section{Conclusiones}

Tenemos dos etapas bien definidas: La etapa actual, cuyo objetivo es desarrollar e implementar un sistema de aislante térmico que aumente en $5^{\circ} \mathrm{C} \circ 6^{\circ} \mathrm{C}$ la temperatura en el interior de las viviendas alto andinas y la segunda etapa, que consiste en masificar el sistema llevando "CONFORT TÉRMICO" a una escala regional, donde se busca replicar lo aprendido en otras comunidades, interviniendo la mayor cantidad posible de hogares (Fig. 12). Para este fin es indispensable la colaboración de diferentes instituciones estatales y privadas para capacitar a las comunidades en las formas en que pueden hacer uso del sistema de manera independiente, mediante talleres dirigidos a los mismos pobladores para que implementen y lo repliquen en otras comunidades, logrando una masificación de este sistema constructivo. Continuaremos visitando comunidades en las alturas, apostando a un ecosistema educativo y experimental, buscando un sueño que nació en las aulas:
Terminar con las muertes producidas por las heladas... Aún queda mucho por hacer

\section{RefERENCIAS}

\section{Burga, J. (2010). Arquitectura vernácula} peruana. Un análisis tipológico. Lima: Colegio de arquitectos del Perú.

Cadillo, K. (2013). ¿Por qué todos los años hay muertos por frío? Velaverde, 18(1). 38-40. Recuperado de https://issuu.com/ revvelaverde/docs/velaverde_edic_18

COEN. (2016). Recomendaciones del COEN para afrontar las heladas y el friaje. El Comercio. Recuperado de https://elcomercio.pe/peru/recomendaciones-coenafrontar-heladas-friaje-417746

República de Perú. Presidencia del Consejo de Ministros. (21 de febrero de 2017). Plan Multisectorial ante Heladas y Friaje 2017. [Decreto Supremo No 019-2017PCM]. Diario el Peruano. Recuperado de http://www.pcm.gob.pe/wp-content/ uploads/2017/02/ds19-2017.pdf

República del Perú. Ministerio del Ambiente, (2014). Huancayo entre las 10 ciudades con más basura. Diario Correo. Recuperado de https://diariocorreo.pe/ciudad/ huancayo-entre-las-10-ciudades-conmas-basura-546352/

SENAMHI. (2003). Proyecto PER/98/G31: Electrificación Rural a base de Energía Fotovoltaica en el Perú. Atlas energía solar del Perú. Lima: Senamhi, República de Perú, UNDP, Ministerio de Energía y Minas. Recuperado de http://cedecap. org.pe/uploads/biblioteca/80bib_arch. pdf 
